\title{
A NOTE ON THE RELATION BETWEEN INTEGRAL AND TCHEBYCHEFF APPROXIMATION BY POLYNOMIALS IN THE COMPLEX DOMAIN*
}

BY W. E. SEWELL

1. Introduction. Let $C$ be a rectifiable Jordan curve in the z-plane and let $R$ be the limited simply connected region bounded by $C$. Let $f(z)$ be analytic in $R$ and continuous in $R+C \equiv \bar{C}$, and let $P_{n}(z)$ be a polynomial of degree $n$ in $z$ which minimizes the integral

$$
\int_{C} w(z)\left|f(z)-P_{n}(z)\right|^{p}|d z|,
$$

where $p$ is a fixed positive number, and $w(z)$ is a bounded nonnegative measurable function bounded from zero; the existence of such a polynomial $P_{n}(z)$ is well known. $\dagger$ Dunham Jackson $\ddagger$ has given an evaluation for $\left|f(z)-P_{n}(z)\right|, z$ in $\bar{C}$, with various restrictions on $C$ and on $R$. In this note we sharpen these results for curves with corners, and extend them to more general smooth curves and to arbitrary rectifiable Jordan curves.

We also consider the case $p=2$ in particular and the development of $f(z)$ of class $L^{2}$ in normal and orthogonal polynomials. Our two principal results are the following theorems.

Theorem A. Let $C$ be a rectifiable Jordan curve in the z-plane and let $f(z)$ be analytic in $C$ and continuous in $\bar{C}$. Let $p_{n}(z)$ be an arbitrary polynomial of degree $n$ such that $\left|f(z)-p_{n}(z)\right| \leqq \epsilon_{n}, z$ in $\bar{C}$. Then $\left|f(z)-P_{n}(z)\right| \leqq M n^{2 / p} \epsilon_{n}, p>0, z$ in $\bar{C}$, where $M$ is $a$ constant independent of $n$ and $z$, and $P_{n}(z)$ is a polynomial of degree $n$ which minimizes (1).

* Presented to the Society, December 31, 1936.

† See, for example, J. L. Walsh, Interpolation and Approximation, Colloquium Publications of this Society, vol. 20, 1935, pp. 351-352.

$\ddagger$ On certain problems of approximation in the complex domain, this Bulletin, vol. 36 (1930), pp. 851-857; On the application of Markoff's theorem to problems of approximation in the complex domain, this Bulletin, vol. 37 (1931), pp. 883890. These papers will be referred to hereafter as JI and JII, respectively. 
TheOREM B. Let $C$ be a rectifiable Jordan curve in the z-plane and let $f(z)$ belong to $L^{2}$ on $C$. Let $\left\{P_{n}(z)\right\}$ be the set of polynomials normal and orthogonal on $C$ and let*

$$
a_{k}=\int_{C} f(z) \overline{P_{k}(z)}|d z|
$$

If $\sum_{k=0}^{\infty}\left|a_{k}\right| k$ converges, the function $f_{1}(z) \equiv \sum_{k=0}^{\infty} a_{k} P_{k}(z)$ is analytic in $C$, continuous in $\bar{C}$, and $f_{1}(z)=f(z)$ almost everywhere on $C$.

The method is an application of recent results of the author $\dagger$ on the modulus of the derivative of a polynomial and is the same as that used by Jackson in JI and JII.

It should be noted here that in $\$ 3$ (Theorem $A$ ) the region may be a multiply connected region bounded by a finite number of rectifiable Jordan curves, or made up of a finite number of separate regions of similar character. $\ddagger$

2. Jordan Curves and Derivatives of Polynomials. § Let $R$, with boundary $C$, be a limited simply connected region in the $z$-plane and let $z=\psi(w)$ map $K$, the complement (with respect to the extended plane) of $\bar{C}$, on $|w|>1$ so that the points at $\infty$ in the two planes correspond to each other. We will say that $C$ is a curve of Type $S \|$ if

$$
\begin{aligned}
0<N_{1}<\left|\frac{\psi\left(w_{1}\right)-\psi\left(w_{2}\right)}{w_{1}-w_{2}}\right| & <N_{2}<\infty, \\
& \left(\left|w_{1}\right| \geqq 1,\left|w_{2}\right| \geqq 1\right),
\end{aligned}
$$

where $N_{1}$ and $N_{2}$ are constants independent of $w_{1}$ and $w_{2}$. If $C$ is a curve of Type $S$ it is shown in SII that for $P_{n}(z)$, an arbitrary polynomial of degree $n$, the inequality $\left|P_{n}(z)\right| \leqq M, z$ on $C$, im-

* $a$ denotes the conjugate of the complex number $a$.

$\dagger$ On the modulus of the derivative of a polynomial, this Bulletin, vol. 42 (1936), pp. 699-702; Generalized derivatives and approximation by polynomials, Transactions of this Society, vol. 41 (1937), pp. 84-123. These papers will be referred to hereafter as SI and SII, respectively.

$\ddagger$ See JII, p. 885.

$\S$ The results given here are extensions of Bernstein's and Markoff's Theorems on the moduli of the derivatives of polynomials; see SII for references.

\| For the geometric properties of $C$ see W. Seidel, Über die Ränderzuordnung bei konformen Abbildungen, Mathematische Annalen, vol. 104 (1931), pp. 182243 ; especially pp. 217-221. 
plies* $\left|P_{n}^{\prime}(z)\right| \leqq M M_{1} n, z$ on $C$; here, and below $M$ and $M_{1}$ are constants independent of $n$ and $z$; the constant $M_{1}$ depends on $C$.

For curves with corners we need the following definition (see SII).

Definition. Let $C$ be a Jordan curve composed of a finite number of Jordan arcs meeting in corners $z_{1}, z_{2}, \cdots, z_{r}$, of exterior openings $\mu_{1} \pi, \mu_{2} \pi, \cdots, \mu_{r} \pi,\left(2>\mu_{1} \geqq \mu_{2} \geqq \cdots \geqq \mu_{r}>0\right)$, and let the difference quotient of the mapping function [see (2)] be bounded in modulus on each sub-arc not containing a corner. Let $t=\mu_{1}$ if $\mu_{1} \geqq 1$, and $t=1$ if $\mu_{1}<1$. Then we shall say that $C$ is a curve of Type $t$.

If $C$ is a curve of Type $t$, the inequality $\left|P_{n}(z)\right| \leqq M, z$ on $C$, implies $\left|P_{n}^{\prime}(z)\right| \leqq M M_{1} n^{t}$, z on $C$ (see SII).

If $C$ is a rectifiable Jordan curve it is shown in SI that $\left|P_{n}(z)\right|$ $\leqq M, z$ on $C$, implies $\left|P_{n}^{\prime}(z)\right| \leqq M M_{1} n^{2}, z$ on $C$.

3. Integral and Tchebycheff Approximation. For approximation in the sense of least $p$ th powers we have the following theorem.

THEOREM 1. Let $C$ be a rectifiable Jordan curve in the z-plane and let $f(z)$ be analytic in $C$ and continuous in $\bar{C}$. Let $P_{n}(z)$, $(n=1,2, \cdots)$, be a polynomial of degree $n$ which minimizes

$$
\int_{C} w(z)\left|f(z)-P_{n}(z)\right|^{p}|d z|
$$

where $w(z)$ is a bounded positive measurable function, with a positive lower bound, and $p$ is a fixed positive number. Let $p_{n}(z)$ be a polynomial of degree $n$ such that $\left|f(z)-p_{n}(z)\right| \leqq \epsilon_{n}$, z in $\bar{C}$. Then we have

$$
\left|f(z)-P_{n}(z)\right| \leqq M n^{t / p} \epsilon_{n}, \quad(z \text { in } \bar{C})
$$

where $M$ is a constant independent of $n$ and $z$, and $t=1$ if $C$ is a curve of Type $S, 1 \leqq t<2$ if $C$ is a curve of Type $t$, and $t=2$ if $C$ is an arbitrary rectifiable Jordan curve.

* $f^{\prime}(z)$ denotes the first derivative of $f(z)$. 
We indicate the proof (see JI). Let $r_{n}(z)=f(z)-p_{n}(z)$, and let $\pi_{n}(z)=P_{n}(z)-p_{n}(z)$; then $r_{n}(z)-\pi_{n}(z)=f(z)-P_{n}(z)$. Let

$$
\begin{aligned}
\gamma_{n} & =\int_{C} w(z)\left|f(z)-P_{n}(z)\right|^{p}|d z| \\
& =\int_{C} w(z)\left|r_{n}(z)-\pi_{n}(z)\right|^{p}|d z| .
\end{aligned}
$$

Let $\left|\pi_{n}(z)\right| \leqq \mu_{n}, z$ on $C$, and let $\left|\pi_{n}\left(z_{0}\right)\right|=\mu_{n}$, where $z_{0}$ is a point of $C$. Then by the results of $\S 2$ we know that $\left|\pi_{n}^{\prime}(z)\right| \leqq \mu_{n} M_{1} n^{t}$, $z$ on $C$, and it follows that

$$
\left|\pi_{n}(z)-\pi_{n}\left(z_{0}\right)\right| \leqq \mu_{n} M_{1} n^{t}\left|z-z_{0}\right|,
$$

$z$ on $C$. Let $s$ be the arc or arcs of $C$ consisting of the set of points $\zeta: \zeta$ on $C,\left|\zeta-z_{0}\right|<1 /\left(2 M_{1} n^{t}\right)$. On $s$ we have $\left|\pi_{n}(\zeta)-\pi_{n}\left(z_{0}\right)\right|$ $\leqq \mu_{n} / 2$. This means that on $s$, whose total length is not less than $1 /\left(M_{1} n^{t}\right)$, we have $\left|\pi_{n}(\zeta)\right| \geqq \mu_{n} / 2$. Let $V \geqq w(z) \geqq v>0$, and we have for $\mu_{n} \geqq 4 \epsilon_{n}$

$$
\gamma_{n} \geqq v\left(\frac{\mu_{n}}{4}\right)^{p} \frac{1}{M_{1} n^{t}}
$$

and by the minimizing property of $P_{n}(z)$, we know that

$$
\gamma_{n} \leqq L V \epsilon_{n}^{p},
$$

where $L$ is the length of $C$. Combining inequalities (4) and (5) yields $\mu_{n} \leqq M_{2} n^{t / p} \epsilon_{n}$, where $M_{2}$ is a constant depending on $C, p$, and $w(z)$, but independent of $n$ and $z$. Thus whether $\mu_{n} \geqq \epsilon_{n}$ or not we have

$$
\left|f(z)-P_{n}(z)\right|=\left|r_{n}(z)-\pi_{n}(z)\right| \leqq M n^{t / p} \epsilon_{n}, \quad(z \text { in } \bar{C}),
$$

where $M$ is independent of $n$ and $z$, and the proof is complete.

Jackson (JI) establishes Theorem 1 with $t=1$ for $C$ satisfying the condition that there is a number $r_{0}>0$ such that at every point of $C$ a circle of radius $r_{0}$ can be drawn tangent to $C$, and containing in its interior and on its boundary only points of $\bar{C} .^{*}$ $\mathrm{He}$ also obtains (JII) the result with $t=2$ for the case where $C$ is a curve such that its parametric representation satisfies a

* For a discussion of such properties see Seidel, loc. cit. 
Lipschitz condition* of order 1 and $R$ is a region for which there is a positive number $r_{0}$ such that from every point of its boundary a line segment of length $2 r_{0}$ can be drawn belonging wholly to the closed region $\bar{R}$.

If $C$ is an analytic Jordan curve and $f^{(j)}(z),(j \geqq 0)$, satisfies $\dagger$ a Lipschitz condition of order $\alpha,(0<\alpha \leqq 1)$, on $\bar{C}$, we know by a theorem of John Curtiss $\ddagger$ that $p_{n}(z)$ exists such that $\epsilon_{n} \leqq M_{1} / n^{j+\alpha}$, where $M_{1}$ is a constant independent of $n$ and $z$. Consequently, in this case we have by inequality (3)

$$
\left|f(z)-P_{n}(z)\right| \leqq \frac{M}{n^{j+\alpha-1 / p}}, \quad(z \text { in } \bar{C}) ;
$$

and hence if $j+\alpha>1 / p$, we have uniform convergence of the sequence $P_{n}(z)$ to $f(z)$ in $\bar{C}$. In fact (6) gives an upper bound on the degree of Tchebycheff approximation of $P_{n}(z)$.

In connection with (3) it is interesting to note that for each $n$ we have§

$$
\lim _{p \rightarrow \infty} P_{n}(z)=T_{n}(z)
$$

where $P_{n}(z)$ is the polynomial of degree $n$ of best approximation to the continuous function $f(z)$ on $C$, a rectifiable Jordan curve, in the sense of least $p$ th powers with a norm function, $\|$ and $T_{n}(z)$ is the polynomial of degree $n$ of best approximation to $f(z)$ on $C$ in the sense $\int$ of Tchebycheff.

* $f(z)$ satisfies a Lipschitz condition of order $\alpha$ on the set $E$ if for arbitrary points $z_{1}$ and $z_{2}$ on $E$ we have $\left|f\left(z_{1}\right)-f\left(z_{2}\right)\right| \leqq L\left|z_{1}-z_{2}\right| \alpha$, where $L$ is a constant independent of $z_{1}$ and $z_{2}$.

$\dagger f^{(0)}(z) \equiv f(z)$.

$\ddagger A$ note on the degree of polynomial approximation, this Bulletin, vol. 42 (1936), pp. 873-878.

$\S$ G. Julia, Sur les polynomes de Tchebycheff, Comptes Rendus (Paris), vol. 182 (1926), pp. 1201-1202; the corresponding result for $C$ the segment $(0,1)$ of the axis of reals is due to G. Polya, Sur un algorithme toujours convergent pour obtenir les polynomes de meilleure approximation de Tchebycheff pour une fonction continue quelconque, ibid., vol. 153 (1915), pp. 840-843.

\| See J. L. Walsh, Approximation by Polynomials in the Complex Domain, Mémorial des Sciences Mathématiques, vol. 73 (1935); especially p. 24.

T That is, $\max \left[\left|f(z)-T_{n}(z)\right|, z\right.$ on $\left.C\right]$ is less than the corresponding expression for any other polynomial of degree $n$. 
4. The Case* $p=2$. Approximation in the sense of least squares leads to a consideration of the set of polynomials $\left\{P_{n}(z)\right\}$ normal and orthogonal on $C$, a rectifiable Jordan curve in the $z$-plane. The method used in proving Theorem 1 serves to establish the following result.

Theorem 2. Let $C$ be a rectifiable Jordan curve in the z-plane and let $\int_{C}\left|Q_{n}(z)\right|^{p}|d z|=\epsilon_{n}, p>0$, where $Q_{n}(z)$ is a polynomial of degree $n$. Then we have

$$
\left|Q_{n}(z)\right| \leqq M n^{t / p} \epsilon_{n}^{1 / p}, \quad \quad(z \text { on } C),
$$

where $M$ is a constant independent of $n$ and $z$, and $t=1$ if $C$ is a curve of Type $S, 1 \leqq t<2$ if $C$ is a curve of Type $t$, and $t=2$ if $C$ is an arbitrary rectifiable Jordan curve.

For the set of polynomials $\left\{P_{n}(z)\right\}$ normal and orthogonal on $C$, we have $\epsilon_{n}=1$ with $p=2$ in Theorem 2 . Thus (7) becomes

$$
\left|P_{n}(z)\right| \leqq M n^{t / 2}, \quad(z \text { on } C) \text {. }
$$

Now suppose $f(z)$ belongs to $L^{2}$ on $C$; then

$$
\begin{gathered}
f(z) \sim a_{0} P_{0}(z)+a_{1} P_{1}(z)+\cdots+a_{k} P_{k}(z)+\cdots, \\
a_{k}=\int_{C} f(z) \overline{P_{k}(z)}|d z|,
\end{gathered}
$$

where the sign $\sim$ is used to denote formal correspondence. The polynomial of degree $n$ of best approximation to $f(z)$ on $C$ in the sense of least squares is $S_{n}(z)=\sum_{k=0}^{n} a_{k} P_{k}(z)$. Now suppose $\sum_{k=0}^{\infty}\left|a_{k}\right| k^{t / 2}$, where the value of $t$ is compatible with the character of $C$, converges, then

$$
f_{1}(z)=\sum_{k=0}^{\infty} a_{k} P_{k}(z)
$$

converges absolutely and uniformly on, and hence within, $C$ and consequently $f_{1}(z)$ is analytic in $C$ and continuous in $\bar{C}$. Moreover, since $S_{n}(z)$ converges in the mean to $f(z)$ on $C$, the function $f_{1}(z)=f(z)$ almost everywhere on $C$. Thus we have the following theorem.

* See G. Szegö, Über orthogonale Polynome, die zu einer gegebenen Kurve der komplexen Ebene gehören, Mathematische Zeitschrift, vol. 9 (1921), pp. 218270 ; J. L. Walsh, Interpolation and Approximation, Chap. VI. 
Theorem 3. Let $C$ be a rectifiable Jordan curve in the z-plane and let $f(z)$ belong to $L^{2}$ on $C$. Let $\left\{P_{n}(z)\right\}$ be the set of polynomials normal and orthogonal on $C$ and let

$$
\begin{gathered}
f_{1}(z)=a_{0} P_{0}(z)+a_{1} P_{1}(z)+\cdots+a_{k} P_{k}(z)+\cdots, \\
a_{k}=\int_{C} f(z) \overline{P_{k}(z)}|d z| .
\end{gathered}
$$

Suppose $\sum_{k=0}^{\infty}\left|a_{k}\right| k^{t / 2},(2 \geqq t \geqq 1)$, converges. Then $f_{1}(z)$ is analytic in $C$, continuous in $\bar{C}$, and is equal to $f(z)$ almost everywhere on $C$ if either: (1) C is a curve of Type $S$ and $t=1$, or (2) $C$ is curve of Type $t$ and $2>t \geqq 1$, or (3) $C$ is an arbitrary rectifiable Jordan curve and $t=2$.

Of course in the above theorem the function $f(z)$ may be defined (or redefined) on a set of measure 0 on $C$ and defined in $C$ so as to coincide everywhere with $f_{1}(z)$.

Georgia School of Technology 